\title{
Actividades colaborativas en el aprendizaje de marcadores discursivos en estudiantes universitarios
}

\section{Actividades colaborativas en el aprendizaje de marcadores discursivos en estudiantes universitarios}

Erika María Villar Revello* iD Universidad Peruana de Ciencias Aplicadas, Lima, Perú ORCID: https://orcid.org/0000-0003-2257-7604

Alejandra Fuerte Tapia (D) Universidad del Valle de México, Toluca, México ORCID: https://orcid.org/0000-0003-4653-9864

Joaquín Vertiz Osores ID Universidad César Vallejo, Lima, Perú

ORCID: https://orcid.org/0000-0002-7606-476X

Eric Gálvez Suárez Universidad Nacional Mayor de San Marcos, Lima, Perú Universidad Privada del Norte, Lima, Perú

ORCID: https://orcid.org/0000-0002-7642-5019

José Antonio Arévalo Tuesta DUniversidad Nacional Federico Villareal, Lima, Perú ORCID: https://orcid.org/0000-0003-0341-7234

Recibido 15-06-18 Revisado 05-07-18 Aprobado 08-10-18 En línea 16-10-18

*Correspondencia

Email: pchuervi@upc.edu.pe

\begin{tabular}{l} 
Citar como: \\
\hline Villar, E., Fuerte, A., Vértiz, J., Gálvez, E., \& \\
Arévalo, J. (2018). Actividades colaborativas \\
en el aprendizaje de marcadores discursivos \\
en estudiantes universitarios. Propósitos y \\
Representaciones 6(2), 607-629. Doi: http:// \\
dx.doi.org/10.20511/pyr2018.v6n2.250
\end{tabular}

(C) Universidad San Ignacio de Loyola, Vicerrectorado de Investigación, 2018

(cc) BY-NC-ND Este artículo se distribuye bajo licencia CC BY-NC-ND 4.0 Internacional (http://creativecommons.org/licenses/by-nc-nd/4.0/). 


\section{Resumen}

El objetivo de la presente investigación es analizar la influencia de las actividades colaborativas en el aprendizaje de los marcadores discursivos en los estudiantes del curso Comprensión y Producción de Lenguaje de una universidad privada de Lima. Es una investigación aplicada con un diseño cuasi experimental, conformándose los grupos, experimental y control. Para la recolección de datos, se empleó la Prueba sobre marcadores discursivos. Asimismo, se efectuó sucesivamente una medición pre test, y obtenidos los resultados, se aplicó el taller sobre actividades colaborativas, compuesto por 7 sesiones, en el grupo experimental, para luego efectuar las mediciones post test, en ambos grupos. La contrastación de hipótesis se efectuó con la prueba U de Mann Whitney. Los resultados mostraron que hubo influencia significativa del trabajo colaborativo sobre el aprendizaje de los marcadores discursivos en los estudiantes objeto de estudio.

Palabras clave: Trabajo colaborativo, aprendizaje, marcadores discursivos. 


\section{Summary}

The objective of this research work is to analyze the influence of collaborative activities on the learning of discourse markers in the students of the Language Comprehension and Production from a private university in Lima. It is an applied research with a quasi-experimental design, composed of experimental and control. For the collection of data, the Test on discourse markers was used. Likewise, a pretest measurement was carried out successively, and once the results were obtained, the workshop on collaborative activities, composed of 7 sessions, was conducted in the experimental group to then carry out the posttest measurements in both groups. The hypothesis testing was made with the Mann Whitney U test. The results showed that there was significant influence of the collaborative work on the learning of the discourse markers in the students that are object of study.

Keywords: Collaborative work, learning, discourse makers. 


\section{Introducción}

En el proceso de redacción es necesario el uso de los marcadores discursivos que, muchas veces, los estudiantes universitarios no emplean. Benavides (2014) señaló que la dificultad que comporta la sistematización de tales unidades lingüísticas no son un solo tipo de palabras; sino que se les asocia a valores relacionados con una categoría gramatical o su ubicación dentro de un enunciado. Así, para estos alumnos universitarios, es muy importante que sepan utilizar los marcadores discursivos en la redacción de textos; siendo necesario plantear estrategias que mejoren este procedimiento.

En la década pasada se tuvieron trabajos interesantes, así Ruiz (2012) determinó la influencia significativa del trabajo cooperativo en el aprendizaje. Errázuriz $(2012,2014)$ señaló que existe una correspondencia entre la adecuación de estos nexos y la progresión lógica de los ensayos. Bonilla (2013) concluyó que el trabajo cooperativo es uno de los modelos que más aportes brinda, pues desarrolla el aprendizaje significativo, coincidiendo con Holgado (2014) y ratificado en trabajos experimentales por Luján (2015), Llontop (2015) y, Serna y Hernández (2016). quienes ya habían señalado que el nivel de estudios si afecta al uso de marcadores, sin embargo a pesar de encontrar una relación directa entre ambos, el nivel de estudios no era determinante, existiendo una tendencia hacia el uso de marcadores argumentativos, de control, de contacto, de modalidad y de cierre (Londoño \& Ospina, 2018).

Definiendo las variables se tuvo que Robledo (2013) sostuvo que las actividades colaborativas se constituyen en "un conjunto de recursos para la organización de los grupos humanos que involucra a los sujetos intervinientes en tal organización, (...), e insta al grupo en pleno a trabajar de forma conjunta para alcanzar metas comunes". (p.31). Asimismo, en lo que respecta a la variable Marcadores discursivos, Martin y Portolés (1999) señalaron que: 
(...) son unidades lingüísticas invariables, que no cumplen un rol sintáctico en el marco de la predicación oracional ni como léxico del español y, tampoco poseen un significado definido; empero, cumplen una función en el discurso o texto: el de conducir, en concordancia con sus características morfosintácticas, semánticas y pragmáticas; a las inferencias que se logran en el proceso comunicativo basado en el uso de una determinada lengua. (p. 110).

Llontop (2015) afirmó que los estudiantes universitarios no son capaces de redactar textos empleando marcadores del discurso, ya que no poseen el conocimiento esperado acerca de cómo estas unidades lingüísticas. Por ello, su redacción no tiene claridad, coherencia y cohesión; ya que son los marcadores discursivos los que les permiten estructurar los textos, esta omisión genera que las producciones escritas no posean fluidez ni cohesión. Esta es la realidad en la que se encuentran muchos estudiantes universitarios en nuestro país, pues carecen de nociones lingüísticas básicas, como el correcto uso de los marcadores discursivos. A nivel local, el estudiante universitario debe demostrar competencias variadas en todos los ámbitos de su vida, ya sea a nivel profesional como personal (Alonso, 2013). Por ello, es importante que sea capaz de leer diversos tipos de textos y que sepa redactar de manera clara, coherente y fluida, utilizando adecuadamente los marcadores discursivos, ya que con estas actividades podrá acceder al conocimiento y desarrollar otras habilidades que le permitirán desenvolverse adecuadamente en este mundo globalizado. Sin embargo, el estudiante a nivel superior demuestra serias deficiencias en estas prácticas, lo que le impide desenvolverse con éxito ante diversas situaciones de escritura. Por tanto, el trabajo colaborativo en el aprendizaje de los marcadores discursivos se presenta como una opción distinta para afrontar las dificultades para redactar, que evidencian los estudiantes universitarios. Este escenario condujo la propuesta que se plasmó en la formulación del problema: ¿Cuál es la influencia de las actividades colaborativas en el aprendizaje de los marcadores discursivos? proponiendo analizar esta influencia en una muestra 
de estudiantes del curso de Comprensión y Producción de lenguaje en una universidad privada en la ciudad de Lima.

\section{Metodología}

Investigación con enfoque cuantitativo, aplicada. Se empleó el método hipotético deductivo (Hernández, Fernández y Baptista, 2014). El diseño fue cuasi experimental, con dos grupos (control y experimental) y dos momentos de mediciones (pre test y post test). La población fueron 350 estudiantes de un curso de Comprensión y Producción de Lenguaje del primer ciclo, de la cual se tomó una muestra de 60 divididos en 30 por cada grupo. Se desarrollaron siete sesiones interventoras. El instrumento que se utilizó fue un test elaborado y validado de 48 ítems, los mismos que tuvieron buena confiabilidad $(\mathrm{KR}=0.860)$. Se midieron cinco dimensiones: Conectores $(9$ ítems); Estructuradores de la información (9 ítems); Reformuladores (12 ítems), Operadores argumentativos (6 ítems) y Marcadores conversacionales (12 ítems). Se tomó en un tiempo máximo de 30 minutos. Para la contrastación de las hipótesis se empleó la prueba $U$ - Mann Whitney $(\alpha=0.05)$.

\section{Resultados}

Previamente, se señala que en las comparaciones entre los grupos control y experimental de la medición pre test no hubieron diferencias significativas, por lo que los resultados inferenciales se emitieron en base a la comparación entre los dos grupos de la medición post test, como se aprecia a continuación. 


\section{Tabla 1.}

Aprendizaje de los marcadores discursivos en los estudiantes del curso de comprensión y producción de lenguaje.

\begin{tabular}{ccccc}
\hline \multirow{2}{*}{ Estadístico } & \multicolumn{2}{c}{ Pretest } & \multicolumn{2}{c}{ Postest } \\
& Control & Experimental & Control & Experimental \\
\hline $\begin{array}{c}\text { Mediana } \\
\text { Desviación cuartil } \\
(\mathrm{QY})\end{array}$ & 27 & 21 & 24 & 35.5 \\
\hline
\end{tabular}

(Pos test: Experimental - control): $U$ de Man-Whitney $=24.500 ; Z=-6.300 ; p=.000$

Se observó que la mediana del grupo experimental fue significativamente mayor que la del grupo control, añadiéndose que la dispersión (QY) fue menor en este grupo, siendo un factor favorable ya que mediante esta intervención no solamente se obtiene mejores resultados en el aprendizaje de los marcadores discursivos sino que se homogeniza el rendimiento del grupo. El detalle se ilustra en la figura 1.

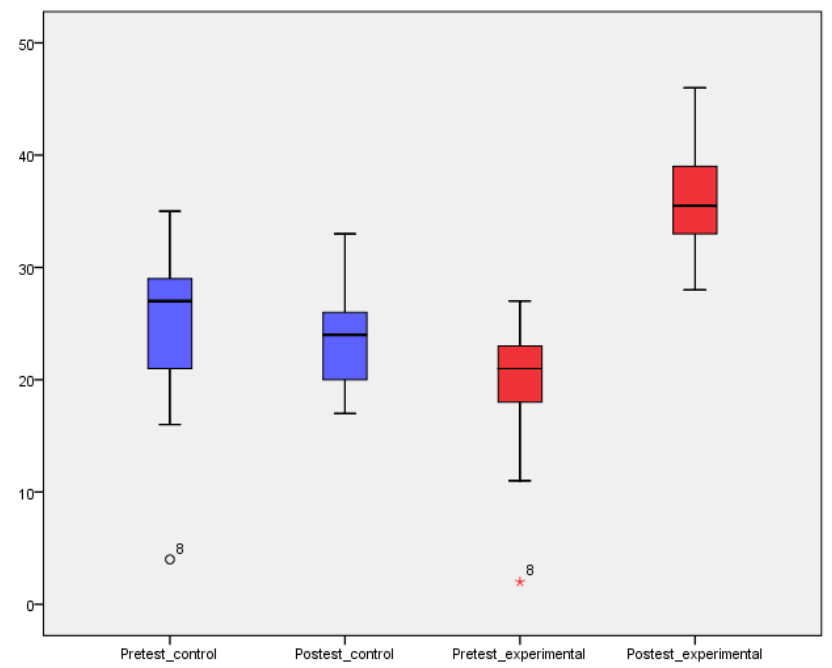

Figura 1. Distribución de los puntajes del Aprendizaje de los marcadores discursivos en los estudiantes del curso de comprensión y producción de lenguaje. 


\section{Tabla 2.}

Aprendizaje de las dimensiones de los marcadores discursivos en los estudiantes del curso de comprensión y producción de lenguaje.

\begin{tabular}{|c|c|c|c|c|c|c|}
\hline \multirow{2}{*}{ Dimensiones } & \multirow{2}{*}{ Estadístico } & \multicolumn{2}{|c|}{ Pretest } & \multicolumn{2}{|c|}{ Postest } & \multirow{2}{*}{$\begin{array}{l}\text { Contraste Postest } \\
\text { (Experimental - } \\
\text { control) }\end{array}$} \\
\hline & & Control & Experimental & Control & Experimental & \\
\hline \multirow{2}{*}{$\begin{array}{l}\text { Aprendizaje de } \\
\text { los conectores }\end{array}$} & Mediana & 5 & 4 & 5 & 7 & $\begin{array}{l}\text { U de Man- } \\
\text { Whitney = }\end{array}$ \\
\hline & QY & 1.47 & 1.18 & 1.41 & 1.17 & $\begin{array}{c}128,500 ; Z= \\
-4.829 ; p=.000\end{array}$ \\
\hline \multirow{2}{*}{$\begin{array}{l}\text { Aprendizaje de los } \\
\text { estructuradores de } \\
\text { la información }\end{array}$} & Mediana & 5 & 4 & 5 & 6 & $\begin{array}{l}\text { U de Man- } \\
\text { Whitney= }\end{array}$ \\
\hline & QY & 1.54 & 1.46 & 1.1 & 1.07 & $\begin{array}{c}98,500 ; Z= \\
-5.322 ; p=.000\end{array}$ \\
\hline \multirow{2}{*}{$\begin{array}{l}\text { Aprendizaje } \\
\text { de los } \\
\text { reformuladores }\end{array}$} & Mediana & 5,5 & 5 & 6 & 9 & $\begin{array}{l}\text { U de Man- } \\
\text { Whitney = }\end{array}$ \\
\hline & QY & 1.89 & 1.65 & 1.41 & 1.27 & $\begin{array}{c}34,000 ; Z= \\
-6.204 ; p=.000\end{array}$ \\
\hline \multirow{2}{*}{$\begin{array}{l}\text { Aprendizaje de } \\
\text { los operadores } \\
\text { argumentativos }\end{array}$} & Mediana & 3 & 2,5 & 3 & 3 & $\begin{array}{l}\text { U de Man- } \\
\text { Whitney = }\end{array}$ \\
\hline & QY & 0.99 & 1.19 & 1.07 & 1.13 & $\begin{array}{c}302,000 ; Z= \\
-2.260 ; p=.024\end{array}$ \\
\hline \multirow{2}{*}{$\begin{array}{l}\text { Aprendizaje de } \\
\text { los marcadores } \\
\text { conversacionales }\end{array}$} & Mediana & 7 & 5 & 5 & 9 & $\begin{array}{l}\text { U de Man- } \\
\text { Whitney = }\end{array}$ \\
\hline & QY & 2.56 & 1.87 & 1.47 & 1.73 & $\begin{array}{c}27,500 ; Z= \\
-6.304 ; p=.000\end{array}$ \\
\hline
\end{tabular}

Se tuvieron contrastes significativos en todas las comparaciones entre las mediciones pre test y post test de las cinco dimensiones, determinando que los valores del post test fueron mayores estadísticamente que los del pre test, significando que hay influencia significativa de las actividades colaborativas en el aprendizaje de los conectores, estructuradores de la información, reformuladores, operadores argumentativos y marcadores conversacionales en los estudiantes del curso comprensión y producción de lenguaje en una universidad privada. Lo interesante es que en la dimensión 'Aprendizaje de los operadores argumentativos' se obtuvieron medianas similares y un QY no tan distante, no obstante debe señalarse que las diferencias de rangos fueron suficientes para la significancia a 0.05 . 


\section{Discusión}

La intervención mediante actividades colaborativas en el aprendizaje de los marcadores discursivos influyó de manera significativa en el aprovechamiento académico de los estudiantes evaluados en el curso de comprensión y producción de lenguaje (CPL), siendo significativo el aprendizaje y aplicación de los conectores, estructuradores de información, reformuladores, operadores argumentativos y de los marcadores conversacionales. Se recomienda que en futuras investigaciones se añada estrategias de medición de aprendizaje cooperativo, midiéndose de manera colectiva, pudiendo emplear herramientas tecnológicas online como auxiliares para dicho fin (Nuñez, Muñoz \& Mihovilovic, 2006)

Al determinar influencia de las actividades colaborativas en el aprendizaje de los marcadores discursivos en los estudiantes del curso de comprensión y producción de lenguaje se definió que la intervención es significativamente útil. Este resultado coincide con Errázuriz (2013) quien fundamentó que los marcadores discursivos juegan un rol importante para el procesamiento de las inferencias y producción de textos, así mismo Portolés (1998) manifestó que permiten organizar la información dentro del texto y señalan la organización informativa de los discursos. Sin embargo, lo hallado por Holgado (2014) resulta importante de considerar debido a que la utilidad del diccionario para que los estudiantes de español permite conocer y manejar un número de marcadores discursivos, ya que los estudiantes que contaban con la ayuda del diccionario utilizaron un mayor número de marcadores, no tanto en variedad como en cantidad, pudiendo ser relevante para este y otros grupos de estudiantes con características similares. Por otra parte, a pesar de la rigurosidad de la medición realizada, es importante indicar que la innovación de la evaluación haría más fina la obtención de resultados. Al respecto, Ruiz, (2012) y García (2017) resaltaron que si los contenidos son aprendidos mediante técnicas cooperativas planificadas y desarrolladas en la aulas, resultando más beneficiosa la eficacia del aprendizaje cooperativo, entonces podría diseñarse una estrategia de evaluación cooperativa de forma 
colectiva. De tal modo que el aprendizaje cooperativo no solo se emplearía como una estrategia con el fin de acrecentar el éxito y los resultados académicos, sino que, además, sería una manera eficaz de promover un ambiente feliz y prosocial en el aula, lo que contribuirá eficientemente en los resultados afectivos y en una mejora significativa en cuanto a las relaciones interpersonales. Aportando con estrategias, Arroyo (2012) y humada (2008) señalaron que la competencia de trabajo colaborativo se adquiere aplicando diferentes fases de la metodología del aprendizaje basado en proyectos, además de fomentar la interacción y la comunicación entre los integrantes, promoviendo el aprendizaje significativo a través de la solución a problemas. La relevancia del reforzamiento de este aprendizaje se soporta en la necesidad de proporcionar herramientas a los estudiantes no solamente para mejorar su rendimiento (García, 2005; Moreano \& Roca, 2014; Llontop, 2015) sino también para evitar la deserción (Ferruci \& Pastor, 2013).

\section{Referencias}

Alonso, P. (2013). La competencia argumentativa a partir del uso de los conectores discursivos, en textos de estudiantes universitarios de primer ingreso del Centro de Estudios Generales (CEG) de la Universidad Nacional. Revista Nuevo Humanismo, 1(1), 73-83. Recuperado de: http://www.revistas.una.ac.cr/index.php/nuevohumanismo/article/ view $/ 5849 / 6017$

Ahumada, L. (2008). El discurso argumentativo de los estudiantes universitarios en los foros de discusión de un campus virtual: análisis del uso de marcadores discursivos. Psicoperspectivas. Individuo y Sociedad, 5(1), 7-22. Recuperado de: http://www.psicoperspectivas.cl/index.php/ psicoperspectivas/article/view/31

Arroyo, G. (2012). Aprendizaje basado en proyectos como estrategia para fomentar el trabajo colaborativo en la educación a distancia. (Tesis de maestría). Universidad Tecnológica de Monterrey, México. 
Benavides, M. (2014). Los marcadores discursivos en el español informal en costa rica: una propuesta de diccionario. (Tesis de maestría). Universidad de Costa Rica, Costa Rica.

Bonilla, S. (2013). Trabajo cooperativo como estrategia didáctica para desarrollar la capacidad de pensamiento autónomo y crítico promoviendo el aprendizaje significativo en los estudiantes del Colegio San Bartolomé. (Tesis de maestría) Universidad de Cuenca, Ecuador.

Errázuriz, M. C. (2012). Análisis del uso de los marcadores discursivos en argumentaciones escritas por estudiantes universitarios. Perfiles educativos, 34(136), 98-117. Recuperado de: http://www.iisue.unam. $\mathrm{mx} /$ perfiles//numeros/2012/136

Errazuriz, M. C. (2014). El desarrollo de la escritura argumentativa académica: los marcadores discursivos. ONOMÁZEIN, 30, $217-236$. Doi: http://dx.doi.org/10.7764/onomazein.30.13

Ferrucci, G., \& Pastor, C. (2013). Desarrollo alcanzado en la redacción académica por los alumnos ingresantes a un curso de Habilidades Lingüisticas Básica de una Universidad Privada de Lima. (Tesis de maestría). Pontificia Universidad Católica del Perú, Lima.

García, M. (2005). Análisis de marcadores discursivos en ensayos escritos por estudiantes universitarios. Letras, 47(71), 33-62.

García, N. (2016). Marcadores discursivos indicadores de cortesía de la variedad mexicana. Un enfoque pluricéntrico para su enseñanza en ELE. EuroAmerican Journal of Applied Linguistics and Languages, 3(1), 2342. Doi: http://dx.doi.org/10.21283/2376905X.4.62

Hernández, R., Fernández, C., \& Baptista, J. (2014). Metodología de la investigación. (6ta ed.) México: Mc Graw Hill Interamericana.

Holgado, A. (2014). Pragmática lingüistica aplicada al español para extranjeros con especial atención a los marcadores discursivos: El diccionario de marcadores discursivos para estudiantes de español como lengua extranjera. (Tesis doctoral). Universidad de Salamanca Salamanca, España.

Llontop, M. C. (2015). Estrategias metacognitivas en la optimización del aprendizaje de los marcadores discursivos en estudiantes del Programa 
de Estudios Básicos de la Universidad Ricardo Palma, año 2015. (Tesis doctoral). Universidad Ricardo Palma, Lima.

Londoño, D., \& Ospina, M. (2018). Comprensión y producción de textos escritos en instituciones de educación superior. Trilogía Ciencia Tecnología Sociedad, 10(18), 183-202. Doi: https://doi. org/10.22430/21457778.671

Luján, K. (2015). Influencia del aprendizaje cooperativo en el desarrollo de habilidades sociales del nivel secundario, área persona, familia y relaciones humanas, Trujillo 2013-2014. (Tesis de grado) Universidad Nacional de Trujillo, Trujilllo.

Martin, M. A., \& Portolés, J. (1999). Los marcadores del discurso. Madrid: España Calpe.

Moreano, R., Roca, M. (2014). Estrategias cognitivas y metacognitivas de composición escrita que se promueven en un curso de redacción en una Universidad Privada de Lima. (Tesis de maestría). Universidad Ricardo Palma, Lima.

Núñez, P., Muñoz, A., \& Mihovilovic, E. (2006). Las funciones de los marcadores de reformulación en el discurso académico en formación. Revista signos, 39(62), 471-492.

Portolés, J. (1998). Marcadores del discurso. Barcelona: Ariel.

Robledo, B. (2013). El trabajo colaborativo en los alumnos de educación primaria. (Tesis de grado). Universidad Pedagógica Nacional de Michoacán, México.

Ruiz, D. (2012). La influencia del trabajo cooperativo en el aprendizaje del área de Economía en la enseñanza secundaria (Tesis doctoral). Universidad de Valladolid, España.

Serna, M., \& Hernández, N. (2016). Los marcadores discursivos en el habla de la ciudad de Pereira (Tesis de maestría). Universidad Tecnológica de Pereira, Colombia. 